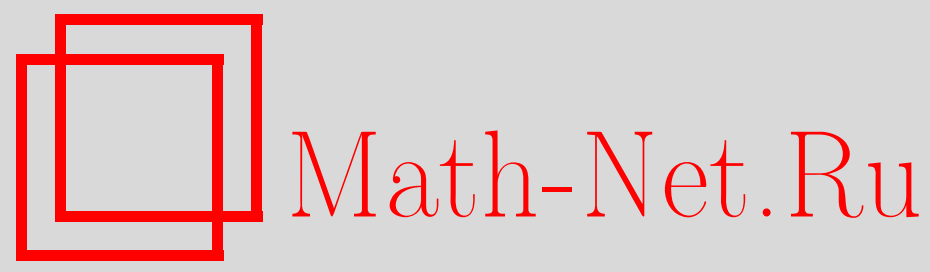

Н. Н. Холщевникова, Теоремы об объединении $U$-множеств, Матем. заметки, 2000, том 67, выпуск 5, 778-787

DOI: https://doi.org/10.4213/mzm895

Использование Общероссийского математического портала Math-Net.Ru подразумевает, что вы прочитали и согласны с пользовательским соглашением http://www.mathnet.ru/rus/agreement

Параметры загрузки:

IP: 54.205 .225 .156

26 апреля 2023 г., 15:10:57

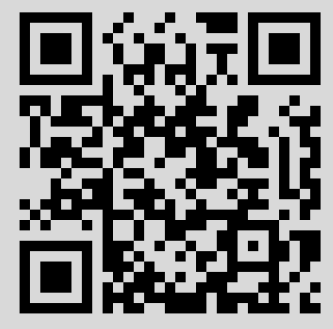




\section{ТЕОРЕМЫ ОБ ОБЪЕДИНЕНИИ $U$-МНОЖЕСТВ}

\section{Н. Н. Холщевникова}

В работе рассматриваются свойства семейств подмножеств топологических пространств, позволяющие выделить некоторое общее ядро доказательства теорем об объединении множеств для рядов по различным системам функций. Как следствие этих результатов, в частности, доказано, что объединение счетного числа замкнутых множеств единственности для тригонометрических рядов является множеством единственности для метода суммирования Абеля-Пуассона.

Библиографоия: 11 названий.

Вопрос об объединении множеств единственности или $U$-множеств: является ли объединение двух (или счетного числа) борелевских множеств единственности снова множеством единственности, возник в 1920-е годы для тригонометрических рядов. Основным результатом здесь является

ТЕОРЕМА БАРИ (см. [1, с. 795]). Сумма конечного числа или счетного множества замкнутых $U$-множеств есть $U$-множество.

В 1986 г. К. Карлет и Г. Дебс (см. [2, с. 44]) получили обобщение этой теоремы, которое мы называем (в связи с его сходностью формулировки и доказательства)

ОБОБШЕННАЯ ТЕОРЕМА БАРИ. Пусть $E_{n}(n \in \mathbb{N})$ последовательность U-множеств, которые замкнуты относительно своего объединения $E=\bigcup_{n=1}^{\infty} E_{n}$. Тогда $E$ тохе U-множество.

Из этой теоремы следуют полученные ранее в [3] результаты: объединение $U$-множества и $U$-множества типа $G_{\delta}$ и $F_{\sigma}$ одновременно и объединение двух непересекающихся $U$-множеств типа $G_{\delta}$ есть $U$-множество.

Для системы Уолша в нумерации Пэли аналог теоремы Бари для объединения конечного числа замкнутых $U$-множеств получил А. А. Шнейдер [4] и в общем случае, для счетного объединения, - В. Вейд [5], а аналог обобщенной теоремы Бари получен в [6].

Можно заметить, что в доказательстве всех этих теорем есть общее ядро.

1. Выделим некоторые свойства, характерные для семейств $U$ - и $M$-множеств, которые позволяют сделать выводы об объединении $U$-множеств в довольно общей ситуации.

Работа выполнена при финансовой поддержке Российского фонда фундаментальных исследований, грант № 99-01-00355. 
Пусть $X$ - топологическоепространство, $U$ - семейство подмножеств $X$, обладающее тем свойством, что

$$
\text { если } A \in U \text { и } A_{1} \subset A \text {, то } A_{1} \in U \text {. }
$$

Такое семейство $U$ назьвается наследственным.

Пусть $M$ - семейство дополнительное $\kappa U$, т. е. семейство множеств, не принадлежащих $U$ :

$$
M=\{A \subset X: A \notin U\}
$$

Назовем множество $A \in M$ ядром, если для всякого открытого множества $G$ такого, что $A \cap G \neq \varnothing$, множество $A \cap G \in M$.

Скажем, что $M-$-емейство со свойством ядра, если каждое множество $E \in M$ содержит подмножество $A$, являющееся ядром.

Замыкание множества $E \subset X$ будем обозначать через $\bar{E}$.

В п. 1 всюду в дальнейшем под $U$ и $M$ понимаются семейства множеств (1) и (2) соответственно топологического пространства $X$.

Лемма 1. Пусть $M$ - семейство со свойством ядра. Если $E_{1}, E_{2} \in U$ u $E_{1}$ замкнуто относительно $E_{1} \cup E_{2}$, то $E_{1} \cup E_{2} \in U$.

ДокаЗАтЕльство. Допустим, что $E_{1} \cup E_{2} \in M$. Так как $M-$ семейство со свойством ядра, то найдется ядро $A$ такое, что $A \subset E_{1} \cup E_{2}$. Тогда

$$
A=\left(A \cap E_{1}\right) \cup\left(A \cap\left(E_{2} \backslash E_{1}\right)\right) .
$$

Так как $E_{1} \in U$ и, следовательно, $A \cap E_{1} \in U$, а $A \in M$, то $A \cap\left(E_{2} \backslash E_{1}\right) \neq \varnothing$. Замкнутость $E_{1}$ относительно $E_{1} \cup E_{2}$ означает, что $\left(E_{1} \cup E_{2}\right) \cap \bar{E}_{1}=E_{1}$. Отсюда следует, что $\left(E_{1} \cup E_{2}\right) \backslash \bar{E}_{1}=E_{2} \backslash E_{1}$.

Положим $G=X \backslash \bar{E}_{1}$. Тогда

$$
A \cap G=A \cap\left(E_{2} \backslash E_{1}\right) \neq \varnothing .
$$

По определению ядра отсюда следует, что $A \cap G \in M$, но это противоречит тому, что $E_{2} \in U$. Следовательно, $E_{1} \cup E_{2} \in U$. Лемма доказана.

СлЕДСТВИЕ. Пусть $M-$ семейство со свойством ядра. Тогда обвединение конечного числа замкнутых множсеств из семейства $U$ принадлежст $U$.

Множество $N$ назовем приведенным ядром ядра $A$, если $N \subset A \subset \bar{N}$ и $N$ не является множеством первой категории на себе.

Лемма 2. Пусть $A-я д р о, N-$ приведенное ядро ядра $A, E_{n} \in U(n=1,2,3, \ldots)$, $E_{n}$ замкнуты относительно своего облединения $E=\bigcup_{n=1}^{\infty} E_{n}$. Тогда $A \backslash E \neq \varnothing$. Если, в частности, $E_{n}$ замкнуты, то и $N \backslash E \neq \varnothing$. 
ДоказАТЕЛьСтво. Допустим, что $A \backslash E=\varnothing$. Тогда

$$
N=\bigcup_{n=1}^{\infty}\left(N \cap E_{n}\right) \text {. }
$$

Так как $N$ не первой категории на себе, одно из множеств $N \cap E_{n}(n=1,2,3, \ldots)-$ множество $N \cap E_{m}$ плотно в $N \cap G \neq \varnothing$ для некоторого открытого множества $G$. Так как $E_{m}$ замкнуто относительно $\bigcup_{n=1}^{\infty} E_{n}$, то $N \cap E_{m}$ замкнуто относительно $N$ и, следовательно, $\varnothing \neq N \cap G \subset E_{m} \cap G$. Положим $G_{1}=G \backslash \bar{E}_{m}$. Тогда

$$
N \cap G_{1}=(N \cap G) \backslash \bar{E}_{m}=\varnothing \text {. }
$$

С другой стороны,

$$
A \cap G_{1}=(A \cap G) \backslash\left(\bar{E}_{m} \cap A\right)=(A \cap G) \backslash\left(E_{m} \cap A\right) \neq \varnothing,
$$

так как $A \cap G \in M$, а $E_{m} \cap A \in U$. Таким образом, $N \cap G_{1}=\varnothing$, а тогда и $\bar{N} \cap G_{1}=\varnothing$, а $A \cap G_{1} \neq \varnothing$. Это противоречит свойству приведенного ядра $A \subset \bar{N}$. Следовательно, $A \backslash E \neq \varnothing$.

Если множества $E_{n}$ замкнуты, то, предполагая, что $N \subset E$, вьполним те же самые построения множеств $G$ и $G_{1}=G \backslash \bar{E}_{m}=G \backslash E_{m}$. Тогда мы опять имеем

$$
N \cap G_{1}=\varnothing, \quad A \cap G_{1}=(A \cap G) \backslash E_{m} \neq \varnothing
$$

и приходим к противоречию (при этом мы теперь не предполагаем, что $A \subset E$ и поэтому замкнутость $E_{n}$ существенна). Лемма доказана.

Назовем $M$ семейством со свойством приведенного ядра, есливсякое ядро $A \in M$ содержит приведенное ядро.

Теорема 1. Пусть $X$ - топологическое пространство, $U$ - наследственное семейство подмножеств $X, M$ - дополнительное $\kappa U$ семейство ядра и со свойством приведенного ядра. Если $E_{n} \in U$ u $E_{n}$ замкнуты относительно своего оббединения $E=\bigcup_{n=1}^{\infty} E_{n}$, то $E \in U$.

ДокАЗАТЕЛЬСтво. Предположим, что $E \in M$. В силу того, что $M-$ семейство со свойством ядра, найдется ядро $A \subset E$, а так как $M$ со свойством приведенного ядра, то $A$ содержит приведенное ядро $N$. Получаем противоречие с леммой 2, в силу которой $A \backslash E \neq \varnothing$. Теорема доказана.

Следствием теоремы 1 является

ТЕорема 2. Пусть $X$ - топологическое пространство, $U$ - наследственное семейство подмножеств $X, M$ - дополнительное $\kappa U$ семейство ядра и со свойством приведенного ядра. Если $E_{n} \in U$ и $E_{n}$ замкнуты $(n \in \mathbb{N})$, то $\bigcup_{n=1}^{\infty} E_{n} \in U$.

Проверка того факта, что $M$ - семейство со свойством ядра, в случае конкретных семейств множеств иногда вызьвает затруднения, и нередко сразу видно, что если объединение счетного числа множеств из $U$ принадлежит $M$, то оно принадлежит подсемейству семейства $M$, каждое ядро которого содержит приведенное ядро. В связи с этим дадим несколько громоздкое, но удобное при использовании обобщение теоремы 1.

Пусть $M_{1}$ подсемейство семейства $M$. Скажем, что $M_{1}$ - подсемейство со свойством ядра, если каждое множество $E \in M_{1}$ содержит ядро, и что $M_{1}$ - подсемейство со свойством приведенного ядра, если всякое ядро $A$ из $M_{1}$ содержит приведенное ядро. 
Теорема $1^{\prime}$. Пусть $X$ - топологическое пространство, $U$ - наследственное семейство подмножеств $X, M$ - дополнительное $\kappa U$ семейство, $M_{1}$ - подсемейство $M$ со свойством ядра и со свойством приведенного ядра. Если $E_{n} \in U, E_{n}$ $(n \in \mathbb{N})$ замкнуты относительно своего оббединения $E=\bigcup_{n=1}^{\infty} E_{n}$ u $E \in U \cup M_{1}$, mo $E \in U$.

ДокаЗАТЕЛЬСтво. Предположение, что $E \in M_{1}$, приводит к противоречию с леммой 2 так же, как в доказательстве теоремы 1 . Теорема доказана.

Далее мы получим некоторые результаты об объединении множеств из $U$ без дополнительных предположений о наличии приведенного ядра.

Лемма 3. Пусть $A \subset E \in M u A$ - ядро. Тогда множество $\bar{A} \cap E$ также является ядром.

ДоКАЗАТЕЛЬСтво. Пусть для некоторого открытого множества $G \bar{A} \cap E \cap G \neq \varnothing$; тогда и $A \cap G \neq \varnothing$ и по определению ядра $A \cap G \in M$, а следовательно, и $\bar{A} \cap E \cap G \in M$. Значит, $\bar{A} \cap E$ также является ядром. Лемма доказана.

Напомним, что топологическое пространство $X$ назьвается полным метризуемым пространством (в топологическом смысле), если на $X$ можно определить метрику, порождающую топологию пространства $X$, и относительно этой метрики $X$ - полноеметрическое пространство. (Так интервал $(0,1)$ - полное метризуемое пространство, хотя как метрическое пространство с естественной метрикой на прямой не является полным.)

Теорема 3. Пусть $X$ - полное метризуемое пространство, $U$ - наследственное семейство подмножеств $X, M$ - дополнительное $\kappa U$ семейство со свойством ядра. Если $E_{n} \in U(n \in \mathbb{N}), E_{n}$ замкнуты относительно своего оббединения $E=\bigcup_{n=1}^{\infty} E_{n}$ и $E$ типа $G_{\delta}$, то $E \in U$. Если, в частности, $E_{n} \in U, E_{n}$ замкнуты u $E$ замкнутое множество, то $E \in U$.

ДоказАтеЛЬСтво. Допустим $E \in M$. Так как $M$ - семейство со свойством ядра, найдется ядро $A \subset B$. В силу леммы $3 \bar{A} \cap E$ также ядро. Если $E$ типа $G_{\delta}$, то ядро $\bar{A} \cap E$ также имеет тип $G_{\delta}$. Но в полном метризуемом пространстве множество типа $G_{\delta}$ не первой категории на себе, поэтому множество $\bar{A} \cap E$ является в то же время приведенньм ядром ядра $\bar{A} \cap E$. Тогда в силу леммы 2 множество $A \backslash \bigcup_{n=1}^{\infty} E_{n} \neq \varnothing$, но это противоречит тому, что $A \subset E$. Теорема доказана.

Теорема 4. Пусть $X$ - полное метризуемое пространство, $U$-наследственное семейство подмножеств $X, M$-дополнительное $\kappa U$ семейство со свойством ядра. Тогда если $E_{1}, E_{2} \in U u$

1) $E_{1}$ и $E_{2}$ множества типа $G_{\delta}$, а $E_{1} \cap E_{2}$ - множество типа $F_{\sigma}$ (в частности, пустое) или

2) $E_{1}$ множество типа $G_{\delta}$ и $F_{\sigma}$ одновременно, то $E_{1} \cup E_{2} \in U$.

ДоказАтельство. Рассмотрим сначала случай 1$)$. Множество $E_{1} \cup E_{2}$ имеет тип $G_{\delta}$. Имеем

$$
E_{1} \cup E_{2}=E_{1} \cup\left(E_{2} \backslash\left(E_{1} \cap E_{2}\right)\right),
$$

причем $E_{2}^{\prime}=E_{2} \backslash\left(E_{1} \cap E_{2}\right)$ тоже имеет тип $G_{\delta}$. Тогда и $E_{1}$, и $E_{2}^{\prime}$ имеют тип $F_{\sigma}$ относительно своего объединения $E_{1} \cup E_{2}$ и, следовательно, $E_{1} \cup E_{2}$ является объединением 
счетного числа множеств, замкнутых относительно $E_{1} \cup E_{2}$ и принадлежащих $U$. Тогда в силу теоремы $3 E_{1} \cup E_{2} \in U$.

Обратимся теперь к случаю 2). Допустим, что $E_{1} \cup E_{2} \in M$. Тогда найдется ядро $A \subset E_{1} \cup E_{2}$. Множество $E_{1} \cap \bar{A}$ имеет тип $F_{\sigma}$, т. е.

$$
E_{1} \cap \bar{A}=\bigcup_{n=1}^{\infty} F_{n},
$$

где $F_{n}$ замкнутые множества. Множество $E_{1} \cap \bar{A}$ имеет тоже тип $G_{\delta}$ и в силу теоремы Бэра о категории $E_{1} \cap \bar{A}$ не первой категории на себе. Отсюда следует, что найдется номер $m$ и открытое множество $G$ такие, что

$$
F_{m} \cap G \text { плотно в } E_{1} \cap \bar{A} \cap G \neq \varnothing,
$$

и так как $F_{m}$ замкнуто, то $F_{m} \cap G=E_{1} \cap \bar{A} \cap G \neq \varnothing$. Положим

$$
A_{1}=E_{1} \cap \bar{A} \cap G, \quad A_{2}=E_{2} \cap G .
$$

Тогда $A_{1}, A_{2} \in U$, множество $A_{1}$ замкнуто относительно $G$, а значит, и относительно $A_{1} \cup A_{2}$. Тогда в силу леммы $1 A_{1} \cup A_{2} \in U$. С другой стороны, так как $\bar{A} \cap G \neq \varnothing$, то и $A \cap G \neq \varnothing$, и так как $A$ - ядро, то $A \cap G \in M$. Но

$$
A \cap G=\left(A \cap E_{1} \cap G\right) \cup\left(A \cap E_{2} \cap G\right) \subset A_{1} \cup A_{2} .
$$

Полученное противоречие доказьвает, что $E_{1} \cup E_{2} \in U$. Теорема доказана.

ЗАмЕЧАнИЕ. Если в условии теоремы 1 в качестве $X$ взять полное метризуемоепространство, то теорема 4 будет следствием теоремы 1. Действительно, в этом случае объединение множеств $E_{1}$ и $E_{2}$, удовлетворяющих условиям 1 ) или 2 ) теоремы 4 , представляется в виде объединения счетного числа замкнутых относительно $E_{1} \cup E_{2}$ множеств из $U$.

2. Рассмотрим теперь применение полученных результатов к решению вопросов об объединении множеств единственности для различных классов рядов.

Пусть $f_{n}$ - отображения топологического пространства $X$ в линейное пространство $Y$ над $\mathbb{R}$ или $\mathbb{C}$. Через $\mathscr{K}$ обозначим класс рядов по системе $\left\{f_{n}\right\}$ вида

$$
\sum_{n} a_{n} f_{n}(x), \quad x \in X
$$

где $a_{n} \in \mathbb{R}$ или $\mathbb{C}$ соответственно, содержащий ряд с $a_{n} \equiv 0$.

Множество $E \subset X$ называется множсеством единственности или $U$-множеством для класса $\mathscr{K}$, коротко $U(\mathscr{K})$-множеством, если из сходимости ряда вида (3) к нулю на множестве $X \backslash E$ следует, что все коэффициенты ряда равны нулю.

Множество $E \subset X$ называется $M(\mathscr{K})$-множеством, если оно не является $U(\mathscr{K})$-множеством.

Семейство $U(\mathscr{K})$-множеств образует, как легко видеть, наследственное семейство $U$, а семейство $M(\mathscr{K})$-множеств - дополнительное к $U$ семейство $M$.

Множество точек, в которых ряд сходится к нулю, назовем множеством нулей ря$\partial a$, д дополнение этого множества - ядром ряда. Приведенным ядром ряда назьвается множество точек, где ряд неограниченно расходится.

Для тригонометрических рядов и для рядов Уолша нуль-рядом называется ряд, которьй почти всюду, но не всюду сходится к нулю.

Для тригонометрических рядов Бари доказана 
Tеорема А (см. [1, с. 794]). Всякая пориия ядра нуль-ряда содержит пориию приведенного ядра того же ряда. Существует другой нуль-ряд, для которого соответственно ядром и приведенным ядром будут служить именно эти пориии ядра и приведенного ядра данного ряда.

Для тригонометрического нуль-ряда приведенное ядро является несчетным множеством типа $G_{\delta}[1$, с. 793$]$.

Отсюда следует, что для тригонометрических рядов семейство $M$-множеств $M$ обладает свойством ядра и приведенного ядра. Действительно, если $E \in M$, то $E$ содержит борелевское подмножество $A \in M$, где $A$ - ядро соответствуюшего ненулевого ряда $S$, сходящегося к нулю вне $E$. Если $m A=0$, то $S$ - нуль-ряд и в силу теоремы А ядро $A$ нуль-ряда $S$ будет ядром семейства $M$, а приведенное ядро нуль-ряда $S$ будет приведенным ядром ядра $A$. Если $m A>0$, то $A$ содержит совершенное подмножество $F$ такое, что если $(a, b) \cap F \neq \varnothing$, то $m((a, b) \cap F)>0$, и так как множество положительной меры является $M$-множеством, то $F$-ядро семейства $M$ и $F$ является в то же время своим приведенным ядром.

Таким образом, справедливость теоремы Бари и обобщенной теоремы Бари для тригонометрических рядов вытекает из теорем 1 и 2.

Аналогично для рядов по системе Уолша семейство $M$-множеств обладает свойством ядра, и свойством приведенного ядра и поэтому аналог теоремы Бари и обобщенной теоремы Бари для рядов по системе Уолша является следствием теорем 1 и 2.

Для системы Фабера-Шаудера, определенной на [0,1],

$$
\varphi_{0}(x)=1, \quad \varphi_{1}(x)=x, \quad x \in[0,1],
$$

и при $n=2^{k}+i\left(k=0,1,2, \ldots ; i=1,2, \ldots, 2^{k}\right)$

$$
\varphi_{n}(x)=\left\{\begin{array}{l}
0, \text { если } x \notin\left(\frac{i-1}{2^{k}}, \frac{i}{2^{k}}\right), \\
1, \text { если } x=\frac{2 i-1}{2^{k+1}}, \\
\text { линейна на }\left[\frac{i-1}{2^{k}}, \frac{2 i-1}{2^{k+1}}\right] \text { и }\left[\frac{2 i-1}{2^{k+1}}, \frac{i}{2^{k}}\right] .
\end{array}\right.
$$

В [7] рассматривался класс $\mathscr{K}$ рядов $\sum_{n=0}^{\infty} A_{n} \varphi_{n}(x)$ со следующим условием на коэффициенты: $A_{n_{k}(z)} \rightarrow 0$ при $k \rightarrow \infty$. Для каждого двоично-рационального $z$, где $\left\{n_{k}(z)\right\}$ - последовательность тех номеров $n$, для которых носитель функции $\varphi_{n}$ (т. е. замыкание множества всех тех $x$, для которых $\varphi_{n}(x) \neq 0$ ) содержит $z$.

Для рядов класса $\mathscr{K}$ в [6] доказано, что непустая порция ядра $A$ ряда, т. е. множество $(a, b) \cap A$, является ядром нового ряда класса $\mathscr{K}$. Отсюда следует, что семейство $M(\mathscr{K})$-множеств $M$ является семейством со свойством ядра. В силу этого для рядов класса $\mathscr{K}$ справедлива теорема 3.

В [7] построено совершенное $M(\mathscr{K})$-множество меры 0. Из теоремы 3 следует, что его нельзя представить в виде объединения счетного числа замкнутых $U(\mathscr{K})$-множеств. При этом заметим, что нуль-ряд по системе Фабера-Шаудера может не иметь точек неограниченной расходимости, т. е. приведенное ядро нуль-ряда может быть и пусто. Для рядов класса $\mathscr{K}$ в [6] доказан аналог теоремы 4 .

Напомним определение обобщенных систем Уолша, введенных Прайсом. 
Пусть $\left\{p_{0}, p_{1}, p_{2}, \ldots\right\}, p_{k} \geqslant 2(k=0,1,2, \ldots)$, последовательность целых чисел. Рассматривается группа $G$, элементами которой являются последовательности целых чисел $x=\left\{x_{0}, x_{1}, \ldots, x_{k}, \ldots\right\}$ такие, что $0 \leqslant x_{k} \leqslant p_{k}-1(k=0,1,2, \ldots)$. По определению групповая операция задается следуюшим образом:

$$
x \dot{+} y=\left\{x_{k}\right\} \dot{+}\left\{y_{k}\right\}=\left\{x_{k} \dot{+} y_{k}\right\}_{k=0}^{\infty},
$$

где $x_{k} \dot{+} y_{k}=x_{k}+y_{k}\left(\bmod p_{k}\right)$.

Подгруппы $G_{n}=\left\{x=\left\{x_{k}\right\} \in G: x_{0}=x_{1}=\cdots=x_{n}=0\right\}$ образуют систему окрестностей нуля, порождающую топологию $G$. По последовательности $\left\{p_{k}\right\}$ определяется последовательность $\left\{m_{n}\right\}$ :

$$
m_{0}=1, \quad m_{n}=\prod_{k=0}^{n-1} p_{k} \quad(n \in \mathbb{N}) .
$$

Любое натуральное $n$ единственным образом представимо в виде $n=\sum_{k=0}^{r} n_{k} m_{k}$, $n_{k}$ - целые, $0 \leqslant n_{k} \leqslant p_{k}-1, k=0,1,2, \ldots$ Обобщенная система Уолша определена на группе $G$ :

$$
\begin{array}{ll}
\psi_{0}(x) \equiv 1, & x \in G, \\
\psi_{n}(x)=\exp \left(2 \pi i \sum_{k=0}^{r} \frac{x_{k} n_{k}}{p_{k}}\right), & x \in G .
\end{array}
$$

При $p_{n} \equiv 2$ получается система Уолша, а при $p_{n} \equiv p(p-$ простое, $p \neq 2)$ - система Крестенсона-Леви.

Для этих обобщенных систем Уолша Н. А. Бокаевым [8] доказаны аналог теоремы Бари и теорема А. Отсюда следует, что для этих систем справедлива теорема 2, т. е. справедлив аналог обобщенной теоремы Бари. А так как топологическая групша $G$ является полным метризуемьм пространством, справедлива также и теорема 4.

Обратимся в заключение к суммированию тригонометрических рядов методом Абеля-Пуассона.

Будем рассматривать тригонометрические ряды

$$
\frac{a_{0}}{2}+\sum_{n=1}^{\infty}\left(a_{n} \cos n x+b_{n} \sin n x\right)
$$

с коэффициентами, стремяшимися к нулю:

$$
\lim _{n \rightarrow \infty} a_{n}=\lim _{n \rightarrow \infty} b_{n}=0 .
$$

Множество $E \subset[0,2 \pi)$ называется множсеством единственности или $U$-множеством для регулярного метода суммирования $T$, если из суммируемости ряда (4), (5) методом $T$ к нулю на $[0,2 \pi) \backslash E$ следует, что $a_{0}=a_{n}=b_{n}=0(n \in \mathbb{N})$.

Всякое множество единственности для регулярного метода суммирования является множеством единственности для тригонометрических рядов. И.И. Привалов [9] доказал, что счетное множество и замкнутое $U$-множество являются множествами единственности для метода суммирования Абеля-Пуассона. 
Напомним, что ряд (4) суммируется в точке $x_{0}$ к числу $s$, если для средних Абеля-Пуассона

$$
f(r, x)=\frac{a_{0}}{2}+\sum_{n=1}^{\infty}\left(a_{n} \cos n x+b_{n} \sin n x\right) r^{n}, \quad 0 \leqslant r<1,
$$

выполняется условие

$$
\lim _{r \rightarrow 1} f\left(r, x_{0}\right)=s .
$$

Если тригонометрический ряд (4), (5) почти всюду, но не всюду суммируется методом Абеля-Пуассона к нулю, то назовем его нуль-рядом для метода Абеля-Пуассона или $\mathscr{A} \mathscr{P}$-нуль-рядом.

Ядром $\mathscr{A} \mathscr{P}$-нуль-ряда назовем множество точек, где он не суммируется к нулю, а приведенным ядром - множество точек, где средние Абеля-Пуассона неограниченны, т. е. $\varlimsup_{r \rightarrow 1}|f(r, x)|=\infty$. Тогда приведенное ядро - это множество

$$
N=[0,2 \pi) \backslash\left\{x: \varlimsup_{r \rightarrow 1}|f(r, x)|<\infty\right\} .
$$

\section{Справедлива следующая}

ЛЕмма 4. Приведенное ядро $N$ для $\mathscr{A} \mathscr{P}$-нуль-ряда $S$ является несчетным множеством типа $G_{\delta}$.

ДокАЗАТЕЛьство. Докажем сначала, что $N$ имеет тип $G_{\delta}$. Пусть

$$
E=[0,2 \pi) \backslash N=\left\{x: \varlimsup_{r \rightarrow 1}|f(r, x)|<\infty\right\} .
$$

Так как коэффициенты ряда $S$ стремятся к нулю, найдется константа $C$ такая, что $|f(r, x)| \leqslant C /(1-r)$. Отсюда следует, что

$$
E=\bigcup_{m=1}^{\infty} E_{m}, \quad \text { где } E_{m}=\{x:|f(r, x)|<m, 0 \leqslant r<1\} .
$$

Так как $E_{m} \subset E_{m+1}$, имеем также $E=\bigcup_{m=1}^{\infty} E_{m+1}$.

Покажем, что $\bar{E}_{m} \subset E_{m+1}$. Действительно, пусть $z=\lim _{k \rightarrow \infty} x_{k}$, где $x_{k} \in E_{m}$. Тогда $|f(r, x)|<m$ для $0 \leqslant r<1$. Зафиксируем $0 \leqslant r<1$. Тогда найдется номер $l=l(r)$ такой, что

$$
\left|\sum_{n=l+1}^{\infty}\left(a_{n} \cos n x+b_{n} \sin n x\right) r^{n}\right|<m+\frac{1}{4} .
$$

Отсюда следует, что

$$
\left|\frac{a_{0}}{2}+\sum_{n=1}^{l}\left(a_{n} \cos n x_{k}+b_{n} \sin n x_{k}\right) r^{n}\right|<m+\frac{1}{4},
$$

а значит, и

$$
\left|\frac{a_{0}}{2}+\sum_{n=1}^{l}\left(a_{n} \cos n z+b_{n} \sin n z\right) r^{n}\right| \leqslant m+\frac{1}{4}
$$


Таким образом,

$$
|f(r, z)| \leqslant m+\frac{1}{2}<m+1
$$

для каждого фиксированного $0 \leqslant r<1$. Следовательно, $z \in E_{m+1}$ и $\bar{E}_{m} \subset E_{m+1}$. Тогда

$$
E=\bigcup_{m=1}^{\infty} E_{m} \subset \bigcup_{m=1}^{\infty} \bar{E}_{m} \subset \bigcup_{m=1}^{\infty} E_{m+1}=E
$$

Значит, $E=\bigcup_{m=1}^{\infty} \bar{E}_{m}$, т. е $E$ имеет тип $F_{\sigma}$. Тогда $N=[0,2 \pi) \backslash E$ имеет тип $G_{\delta}$.

Покажем, что $N$ несчетно. Если бы $N$ было счетньм, то ряд $S$, почти всюду суммирующийся к нулю, имел бы неограниченные при $r \rightarrow 1$ средние $f(r, x)$ разве лишь на счетном множестве $N$ и тогда в силу известной теоремы (см. [10, с. 557]) - аналога теоремы Валле-Пуссена о единственности представления функции тригонометрическим рядом для метода Абеля-Пуассона, $S$ был бы рядом Фурье нулевой функции, что противоречит тому, что $S-\mathscr{A} \mathscr{P}$-нуль-ряд. Таким образом, $N$ несчетно и лемма доказана.

Для доказательства аналога теоремы А обратимся к формальным произведениям тригонометрических рядов.

Формальным произведением ряда (4), которьй в комплексной форме записьвается в виде

$$
\sum_{n=-\infty}^{\infty} c_{n} e^{i n x}, \quad \text { где } \quad c_{-n}=\bar{c}_{n}, \quad c_{n}=\frac{a_{n}-i b_{n}}{2},
$$

и тригонометрического ряда

$$
\sum_{n=-\infty}^{\infty} \gamma_{n} e^{i n x}
$$

назьвается ряд

$$
\sum_{n=-\infty}^{\infty} K_{n} e^{i n x}, \quad \text { где } K_{n}=\sum_{p=-\infty}^{\infty} c_{p} \gamma_{n-p}
$$

Известно (результаты А. Райхмана, см., например, [1, с. 194]), что если $c_{n} \rightarrow 0$ и ряд $\sum\left|\gamma_{n}\right|$ сходится, то $K_{n} \rightarrow 0$. При этом ряд (8) является рядом Фурье своей суммы $\lambda(x)$. Если $c_{n} \rightarrow 0$ и ряд $\sum\left|n \gamma_{n}\right|$ сходится, то ряд

$$
\sum_{n=-\infty}^{\infty}\left(K_{n}-\lambda(x) c_{n}\right) e^{i n x}=\sum_{n=-\infty}^{\infty} K_{n} e^{i n x}-\lambda(x) \sum_{n=-\infty}^{\infty} c_{n} e^{i n x}
$$

сходится к нулю равномерно на $[0,2 \pi)$.

Условие $\sum\left|n \gamma_{n}\right|<\infty$ вьполняется, в частности, если $\sum \gamma_{n} e^{i n x}$ есть ряд ции $\lambda(x)$, имеюший три непрерывные производные.

Справедлив аналог теоремы А.

Лемма 5. Пусть $S-\mathscr{A} \mathscr{P}$-нуль-ряд, $A-$-ео ядро, а $N$ - приведенное ядро. Если

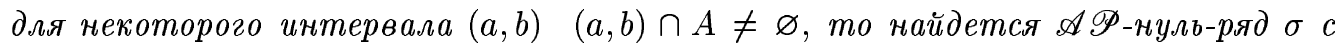
ядром $A \cap(a, b)$ и приведенным ядром $N \cap(a, b)$. 
ДокАЗАТЕЛЬСтво. Рассмотрим ряд $\sigma$, представляющий собой формальное произведение (9) ряда $S$ на ряд Фурье функции $\lambda(x)$, имеющей три непрерывные производные, равные нулю на $[0,2 \pi) \backslash(a, b)$ и положительные на $(a, b)$. В силу (10) метод Абеля суммирует функциональный ряд $\sigma-\lambda(x) \cdot S$ к нулю на $[0,2 \pi)$. Это означает, что

$$
\lim _{r \rightarrow 1}(g(r, x)-\lambda(x) f(r, x))=0, \quad x \in[0,2 \pi),
$$

где $g(r, x)$ и $f(r, x)$ - средние Абеля-Пуассона рядов $\sigma$ и $S$ соответственно. Из (11) следует, что ряд $\sigma$ имеет ядро $A \cap(a, b)$ и приведенное ядро $N \cap(a, b)$. Лемма доказана.

Пусть теперь $U$ - семейство $U$-множеств для метода Абеля-Пуассона, а $M$ - дополнительное к $U$ семейство, т. е. семейство $M$-множеств для метода Абеля-Пуассона. Рассуждение, аналогичное тому, что было приведено после теоремы А, показьвает, что в силу леммы $5 M$ является семейством со свойством ядра и со свойством приведенного ядра. Следовательно, семейства $U$ и $M$ удовлетворяют условиям теорем 1 и 2 . Так как замкнутое $U$-множество является $U$-множеством для метода Абеля-Пуассона, отсюда, в частности, следует

Tеорема 5. Всякое множество единственности типа $F_{\sigma}$ для тригонометрических рядов является также множеством единственности для метода Абеля-Пуассона.

Так как метод Абеля сильнее метода средних арифметических $(C, 1)$, из теоремы 5 вытекает, что всякое $U$-множество типа $F_{\sigma}$ является $U$-множеством и для метода $(C, 1)$. В [11] аналог теоремы 5 получен для метода суммирования Римана.

\section{СПИСОК ЦИТИРОВАННОЙ ЛИТЕРАТУРЫ}

[1] Бари Н. К. Тригонометрические ряды. М.: Физматгиз, 1961.

[2] Kechris A., Louveau A. Descriptive Set Theory and the Structure of Sets of Uniqueness. Cambridge: Cambridge University Press, 1987.

[3] Холщевникова Н. Н. О сумме меньше континуума замкнутых $U$-множеств // Вест. МГУ . Сер. матем., мех. 1981. № 1. С. 51-55.

[4] Шнейдер А. А. О единственности разложений по системе функций Уолша // Матем. сб. 1949. Т. 24 (66). №2. C. 279-300.

[5] Wade W. R. Summing closed $U$-sets for Walsh series // Proc. Amer. Math. Soc. 1971. V. 29. № 1. P. 123-125.

[6] Холщевникова Н. Н. Обобщенная теорема Бари для системы Уолша // Матем. сб. 1992. T. 183. №10. C. $3-12$.

[7] Холщевникова Н.Н. О множествах единственности для рядов по различным системам функций // Изв. РАН. Сер. матем. 1993. Т. 57. №1. С. 167-182.

[8] Бокаев Н. А. О сумме замкнутых $U$-множеств для мультипликативных ортонормированных систем // Вест. МГУ. Сер. матем., мех. 1985. №6. С. 93-96.

[9] Привалов И. И. Обобщение теоремы Paul-du-Boys-Reymond'a // Матем. сб. 1923. Т. 31. № 2 .

[10] Зигмунд А. Тригонометрические ряды. Т. 1. М.: Мир, 1965.

[11] Холщевникова Н. Н. К теореме Валле-Пуссена о единственности представления функции тригонометрическим рядом // Матем. сб. 1996. Т. 187. №5. С. 143-160. 